\title{
99mTC-HMPAO perfusion SPECT/CT in the diagnosis of brain death
}

Thorsten Derlin, Desiree Weiberg

Department of Nuclear Medicine, Hannover Medical School, Hannover, Germany

[Received 27 XII 2015; Accepted 18 VIII 2016]

\begin{abstract}
This report describes a case of brain death (BD) evaluated by ${ }^{99 m}$ Tc-hexamethylpropylene amine oxime (HMPAO) single photon emission tomography/computed tomography (SPECT/CT). A 16-year-old boy with a history of rapid unexpected brain herniation due to pilocytic astrocytoma underwent ${ }^{99 \mathrm{~m} T C-H M P A O ~ S P E C T / C T ~ f o r ~ e v a l u a t i o n ~ o f ~ b r a i n ~ d e a t h ~ i n ~ t h e ~ c o n t e x t ~ o f ~ o r g a n ~ d o n a t i o n . ~}$ Flow images demonstrated lack of blood flow to the brain, and delayed images showed absence of demonstrable radionuclide activity within the brain. SPECT/CT confirmed absence of tracer accumulation, and was deemed helpful for evaluation of the brain stem. ${ }^{99 \mathrm{~m} T C-H M P A O ~ S P E C T / C T ~ i s ~ a ~ v a l u a b l e ~ t o o l ~ e n a b l i n g ~ i m a g i n g-b a s e d ~ c o n f i r m a t i o n ~ o f ~ B D . ~}$
\end{abstract}

KEY words: brain death; perfusion imaging, HMPAO, SPECT, SPECT/CT

Nucl Med Rev 2016; 19, Suppl. B: B22-B23

\section{Background}

Determination of brain death in children is a clinical diagnosis based on the absence of neurologic function with a known irreversible cause of coma [1]. Ancillary studies (such as electroencephalogram and radionuclide cerebral blood flow studies) are not required to establish the diagnosis of brain death, but may be used to assist in establishing the diagnosis, particularly when components of the clinical examination or apnea testing cannot be completed safely due to the underlying medical condition of the patient, when there is uncertainty about the results of the neurologic examination, if a medication effect may be present, or to reduce the observation period before organ donation [1-4]. Brain perfusion scintigraphy using ${ }^{99 m} \mathrm{Tc}-\mathrm{HMPAO}$ represents a well-studied method for diagnosing brain death [5]. Brain perfusion imaging has a very high sensitivity while the specificity (absent cerebral perfusion with clinical confirmation of brain death) is virtually $100 \%$ [5-9].

\section{Case report}

A 16-year-old boy experienced abrupt onset of headaches, dizziness, nausea and finally loss of consciousness. Computed tomography demonstrated acute hydrocephalus occlusus, generalized brain edema, and consecutive brain herniation due to a large infratentorial tumor. The patient underwent craniectomy and tumor

\section{Correspondence to: Thorsten Derlin, MD}

Hannover Medical School, Department of Nuclear Medicine

Carl-Neuberg-Str. 1, D-30625 Hannover, Germany

Tel: 49 (0) 511532 2577; Fax: 49 (0) 5115323761

E-mail: Derlin.Thorsten@mh-hannover.de resection, and histopathologic evaluation later revealed pilocytic astrocytoma. Intraoperative assessment showed extensive brain damage and the intracranial pressure was at $50 \mathrm{mmHg}$. Before organ donation, ${ }^{99 m} \mathrm{Tc}$-hexamethylpropylene amine oxime (HMPAO) single photon emission tomography/computed tomography (SPECT/CT) was performed as an ancillary test for evaluation of brain death (Figure 1). Flow images demonstrated lack of blood flow to the brain, and the sagittal sinus could not be noted. Delayed planar images in anterior and right lateral views showed absence of demonstrable radionuclide activity within the brain. The maximum-intensity-projection SPECT image showed foci of tracer uptake corresponding to the sphenoidal sinuses, and to the parotid and lacrimal glands. SPECT/CT confirmed absence of tracer accumulation, consistent with brain death, and was deemed particularly helpful for evaluation of the brain stem and differentiation of brain stem activity from sinus uptake.

\section{Discussion}

SPECT has been suggested to be helpful for reducing the effect of overlying scalp activity and for evaluating the posterior fossa in the diagnostic work-up of brain death $[8,9]$. In recent years, the introduction of SPECT/CT has improved diagnostic algorithms in many areas of nuclear medicine. The morphological information from CT has been shown to provide useful anatomical details, to improve the reliability of imaging-based diagnoses, to provide data for attenuation correction and - more recently — to provide absolute quantification of uptake in SPECT studies $[10,11]$. Like in this case, SPECT/CT in the context of brain death providing detailed anatomical information could potentially help differentiate brain stem perfusion from other causes of tracer uptake, e.g., adjacent sinus activity, with great reliability. This is the first case in the litera- 

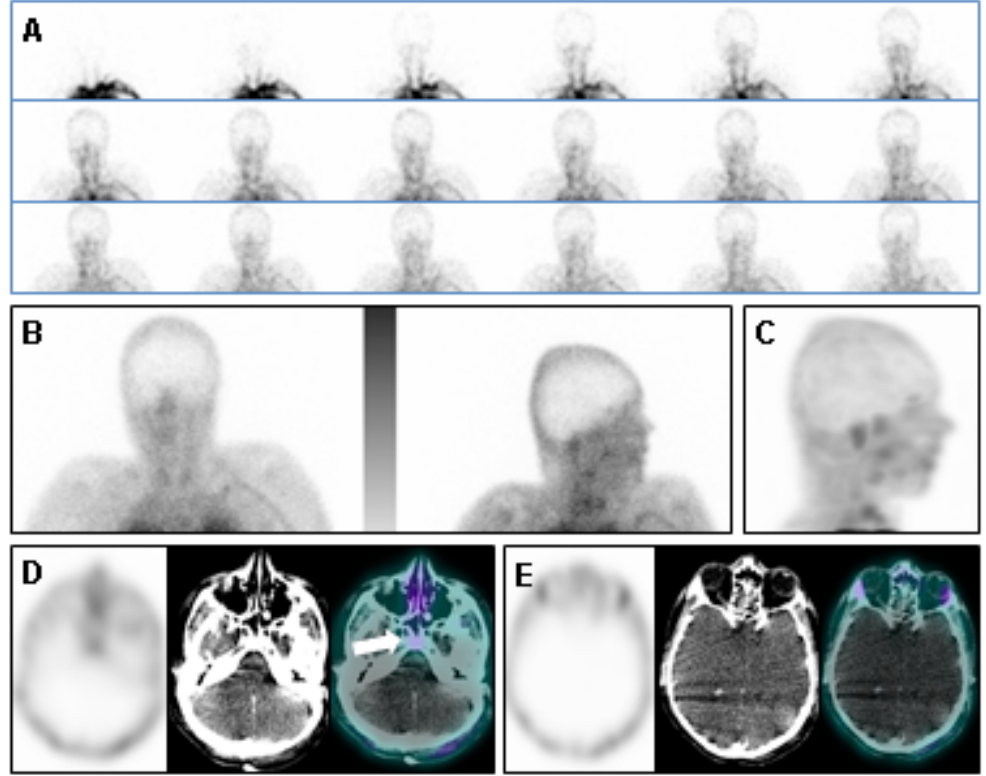

Figure 1A-E. Flow images in the anterior view (A) demonstrated lack of blood flow to the brain, and the sagittal sinus could not be noted. Delayed planar images in anterior and right lateral views (B) showed absence of demonstrable radionuclide activity within the brain. The maximum-intensity-projection SPECT image showed foci of tracer uptake corresponding to the sphenoidal sinuses, and to the parotid and lacrimal glands (C). SPECT/CT at the level of the brain stem (D) and lacrimal glands $(E)$ confirmed absence of tracer accumulation, consistent with brain death, and was deemed particularly helpful for evaluation of the brain stem and differentiation of brain stem activity from sinus uptake (arrow)

ture demonstrating that ${ }^{99 m} \mathrm{TC}-\mathrm{HMPAO}$ SPECT/CT is a valuable tool enabling imaging-based confirmation of brain death.

\section{Conflict of interest}

The authors declare that they have no conflict of interest.

\section{References}

1. Nakagawa TA, Ashwal S, Mathur M et al. Clinical report. Guidelines for the determination of brain death in infants and children: an update of the 1987 task force recommendations. Pediatrics 2011; 128: e720-e740.

2. Greer DM, Varelas PN, Haque S et al. Variability of brain death determination guidelines in leading US neurologic institutions. Neurology 2008; 70 : 284-289

3. Wijdicks EF, Varelas PN, Gronseth GS et al. Evidence-based guideline update: determining brain death in adults: report of the Quality Standards Subcommittee of the American Academy of Neurology. Neurology 2010; 74: 1911-1918
4. Youn TS, Greer DM. Brain death and management of a potential organ donor in the intensive care unit. Crit Care Clin 2014; 30: 813-831.

5. Sinha P, Conrad GR. Scintigraphic confirmation of brain death. Semin Nucl Med 2012; 42: 27-32.

6. Joffe AR, Lequier L, Cave D. Specificity of radionuclide brain blood flow testing in brain death: case report and review. J Intensive Care Med 2010; 25: 53-64.

7. Flowers WM Jr, Patel BR. Radionuclide angiography as a confirmatory test for brain death: a review of 229 studies in 219 patients. South Med J 1997: 90: 1091-1096.

8. Okuyaz C, Gücüyener K, Karabacak NI et al. Tc-99m-HMPAO SPECT in the diagnosis of brain death in children. Pediatr Int 2004; 46: 711-714.

9. Munari M, Zucchetta P, Carollo $\mathrm{C}$ et al. Confirmatory tests in the diagnosis of brain death: comparison between SPECT and contrast angiography. Crit Care Med 2005; 33: 2068-2073.

10. Lange C, Seese A, Schwarzenböck S CT-based attenuation correction in I-123-ioflupane SPECT. PLoS One 2014; 9: e108328.

11. Li S, Sinusas AJ, Dobrucki LW et al. New approach to quantification of molecularly targeted radiotracer uptake from hybrid cardiac SPECT/CT: methodology and validation. J Nucl Med. 2013; 54: 2175-2181. 